\title{
Genetic Status
}

National Cancer Institute

\section{Source}

National Cancer Institute. Genetic Status. NCI Thesaurus. Code C19743.

the state of a person's genome at a given time. 